\title{
Practical Experience in Setting Transformer Differential Inrush Restraint
}

\author{
Rich Hunt \\ GE Multilin
}

\author{
Joe Schaefer \\ Florida Power \& Light Company
}

\author{
Bob Bentert \\ Florida Power \& Light Company
}

\section{Abstract}

The second harmonic inrush restraint function of transformer differential relays maintains security of the differential protection during transformer inrush events. The typical setpoint for the second harmonic restraint is the relay manufacturer's default or recommended setting of $20 \%$ of fundamental current, with some adjustment based on operating experience. However, some operating situations may result in levels of second harmonic current lower than $20 \%$ during inrush, and levels may be as low as $5 \%$. This lower level of second harmonic current requires a lower inrush restraint setting that may impact the tripping time of the differential element for fault conditions. In addition, inrush restraint is typically performed on a per-phase basis, so a loss of security is possible if inrush restraint performs incorrectly on only one phase of the protected transformer.

This paper provides several examples of actual events where loss of security occurred due to incorrect settings of the second harmonic restraint function, or due to mis-application of crossphase blocking. Based on the information from these events, the paper directly discusses considerations and recommendations for setting the second harmonic restraint to maintain security during transformer inrush including a discussion of traditional and adaptive inrush restraint techniques. The paper also includes recommendations on when to apply cross-phase blocking techniques such as 1-out-of-3 blocking, 2-out-of-3 blocking and average restraint blocking methods.

A key message from this paper is the use of the actual inrush characteristic of the protected transformer to determine optimum setpoints for the differential relay. Microprocessor relays have the capability to, and should, capture waveforms every time a transformer is energized. This data should be analyzed to check the adequacy of the existing second harmonic restraint settings, to ensure no loss of security occurs.

\section{Introduction}

Florida Power \& Light Company (FPL) is in the process of replacing existing transformer protection panels at select locations on the FPL transmission system. The existing panels use electromechanical or solid-state differential relays, and the new transformer protection panels will use microprocessor relays with a standard configuration. As part of the design process for these protection panels, FPL is also developing a standard for transformer protection settings.
The basic protection for these transformers is differential protection. Second harmonic restraint is used to block the differential element during inrush events for the transformer. The standard protection settings will include a recommendation for the selection of the inrush restraint function and the settings for this function. Also, these settings will determine whether to use the inrush restraint function on a per-phase or multi-phase basis.

To develop the standard settings for the inrush restraint function, FPL used an experimental laboratory procedure along with actual operating experiences. A relay was configured to some inrush restraint function, level setting, and cross-phase blocking method. These settings were then tested against simulated and actual fault events, by playing oscillographic records back through a test set to the relay. These tests were repeated using different setpoints and different restraint functions until a standard setting that meets FPL's operating criteria was determined. This standard package of settings was confirmed by capturing transformer in-rush records from various installations and comparing the setpoint levels to actual second harmonic levels to confirm there is enough margin.

One of the drivers for this process is an effort to eliminate misoperations of transformer differential relays due to low second harmonics on inrush without sacrificing protection capabilities. Misoperations can occur during the energization of a transformer due to failure of the harmonic restraint function. A digital fault recorder oscillographic record that captures this type of harmonic restraint failure was used for the testing later in this paper. This fault record shows a failure due to the low levels of harmonic current produced during an energization. An external fault can also trigger a misoperation during the voltage recovery period, causing the differential relay to operate immediately after a fault is cleared from the system.

\section{Review of differential protection principles}

Differential protection is a fast, selective method of protection against short circuits in transformers, and is the standard protection used by FPL to protect transformers. Differential protection is a practical application of Kirchhoff's current law. The sum of the currents entering the transformer should equal the sum of the currents leaving the transformer. Differential protection adds the measured currents entering and leaving the transformer to create a differential current. 


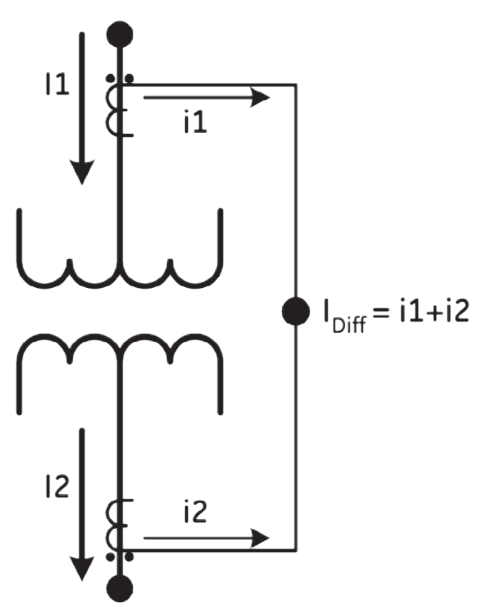

Differential principle

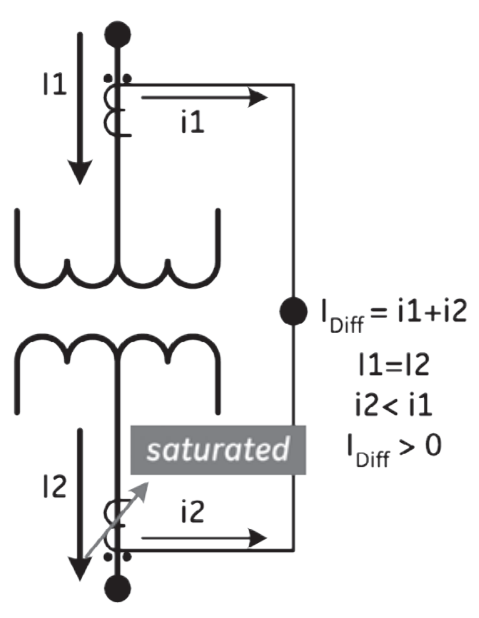

Differential during CT saturation

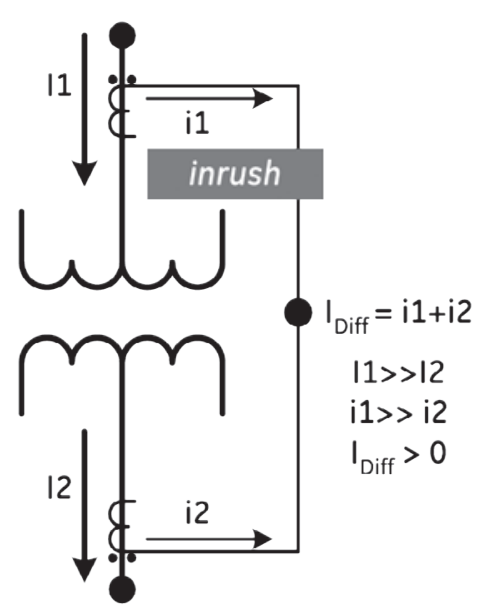

Differential during transformer inrush

Figure 1.

Transformer Differential Protection Principle

With the ideal transformer of Figure 1 , and assuming ideal CTs, the differential current is zero when current is flowing through the transformer. A differential current greater than zero indicates an internal fault condition. In practice, the differential current for a normally operating transformer is always greater than zero due to $\mathrm{CT}$ measurement error, the position of the load tap changer, and other factors introducing noise into the measurement signals. Therefore, the sensitivity of the protection is reduced slightly to account for these errors.

There are two common situations where differential protection may incorrectly declare an internal fault condition. One condition is CT saturation for a fault outside of the transformer zone of protection. The error in the measurement signal of the saturated CT results in a significant error in the differential current. The erroneous differential current may result in undesired operation of the differential element for an external fault condition. This type of event is beyond the scope of this paper.

The second common situation is a transformer inrush event. Some operating situations instantly change the operating flux of the transformer core, requiring a large supply of current. This inrush of current typically occurs in only one winding of the transformer. Therefore inrush currents may produce a differential current that results in the operation of the differential protection. This type of event is not a fault condition, so the differential protection should restrain from operating for this condition.

\section{Transformer Inrush Phenomena}

To properly set a protection function, it is necessary to have a basic understanding of the power system events the function is intended to detect. To set the inrush restraint function for transformer differential protection requires some understanding of transformer inrush events, including the causes and characteristics of these events. This section of the paper defines a transformer inrush event. The section continues on to discuss how power system conditions influence the severity and characteristic of the inrush event, and finishes by describing the common power system events that cause transformer inrush.

\subsection{Definition of Magnetizing Inrush Current}

A transformer inrush event is actually magnetizing inrush current. The windings in a transformer are linked magnetically by the flux in the transformer core. The exciting voltage drives the flux in the core. An increase in the exciting voltage therefore increases the flux. To maintain this additional flux, which may be in the saturation range of the core steel of the transformer, the transformer draws more current which can be in excess of the full load rating the transformer windings. This additional current is the inrush current necessary to supply the magnetizing branch of the transformer. [1]

To show magnetizing inrush current graphically, consider the equivalent circuit of transformer shown in Figure 2. In an ideal transformer (with a 1:1 turns ratio), the currents 11 and 12 are equal except for the small current flowing through the shunt element of the magnetizing branch. The increase in flux caused by an increase in the exciting voltage draws more current through the magnetizing branch. When the transformer is being energized, this current flows through only one winding. In this example, the current $I 1$ is the inrush current. During inrush events other than energization, the magnetizing inrush current may appear in both windings, with the inrush current more prevalent in one winding. Remembering the differential current is, then in any inrush event, the magnetizing inrush current results in a differential current. This differential current can lead to operation of the differential protection. Figure 3 is an example of magnetizing inrush current and the resulting differential current.

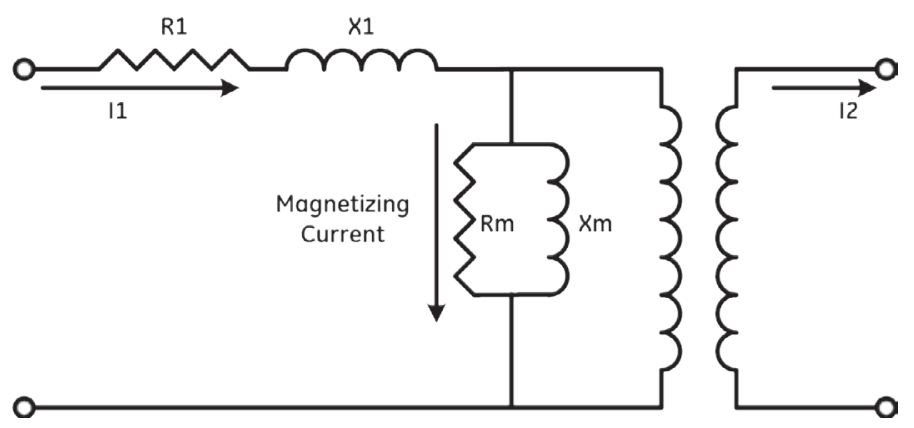

Figure 2.

Transformer Equivalent Circuit 


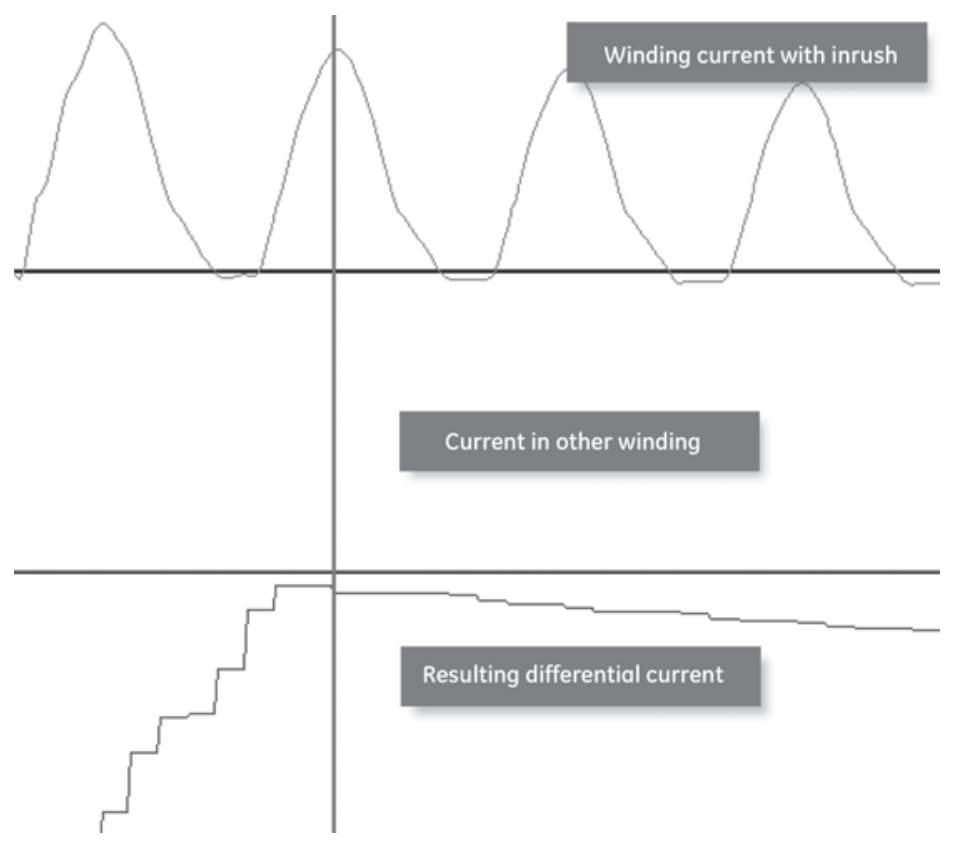

Figure 3.

Inrush Current and Resulting Differential Current

A review of $A C$ excitation of magnetic materials helps understand the actual characteristic of magnetizing inrush current. The magnetic steel used in transformers has a large number of regions (known as "domains") with a specific magnetic moment. An external magnetizing force causes all the magnetic moments of the steel to align with the applied magnetic field. In the case of transformers, the excitation voltage provides this applied magnetic field. The alignment of the magnetic moments causes an increase in flux density greater than that of the external magnetic field. The steel is fully saturated when all the magnetic moments are aligned with the applied field. Once the external field is reduced, the magnetic moments maintain a net magnetization component along by the direction of the field. This effect results in magnetic hysteresis of the steel.[2] Transformers use grain-oriented electrical steel, where the domains tend to produce directions of magnetization with high permeability and low core loss.

Figure 4 shows the exciting voltage e, the core flux $\varphi$, and the exciting current i $\varphi$, of a transformer. The figure also shows the flux and exciting current mapped to the corresponding magnetic hysteresis loop. The excitation voltage drives the flux in the core.

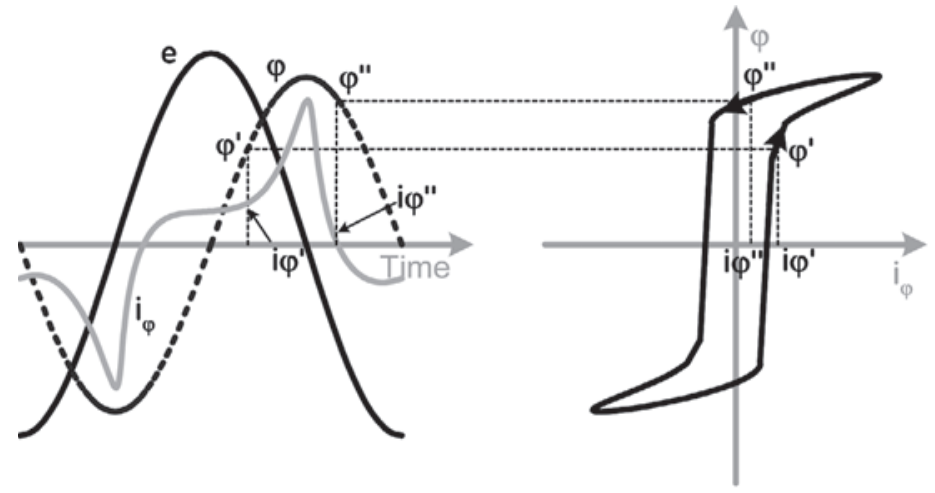

Figure 4.

Transformer Core Excitation Phenomena
The exciting current is needed to produce the magnetic field. The waveform of the exciting current varies from the sinusoidal waveform of the flux due to the non-linear magnetic properties of the core.[2]

The waveforms and hysteresis loop shown in Figure 4 are typical for a transformer that is in service supplying load. The flux requirement is very small, therefore, the exciting current is very small. Now consider what happens when the excitation voltage increases. This voltage drives an increase in the flux in the core. The flux characteristic is still sinusoidal in shape. The flux may be shifted in respect to the 0-axis due to the point on the wave when the excitation increases and the amount of remanent flux in the core. This flux may be high enough to cause saturation of the transformer core. The hysteresis loop becomes negligible for this case, as shown in Figure 5. The resulting current, the magnetizing inrush current, needed to supply the flux is very high in magnitude, and may approach the magnitude of fault currents. The magnetizing current will eventually decay due to losses in the circuit.[3]

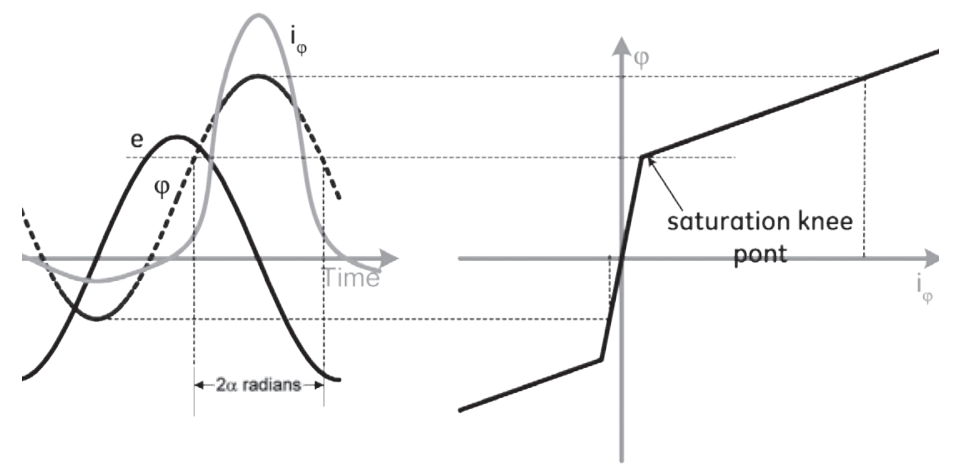

Figure 5.

Flux and Exciting Current Hysteresis during Core Saturation

When the transformer core is in saturation, the exciting current is part of a sine wave for the period that the flux exceeds the saturation knee point of the core. The exciting current is essentially zero for the rest of the power system cycle. This results in the classic waveform signature of magnetizing inrush current, as shown in Figure 6.

The magnitude and characteristic of the inrush current is dependent on the amount of saturation of the transformer core. There are several factors that influence the likelihood the transformer core will go into saturation.

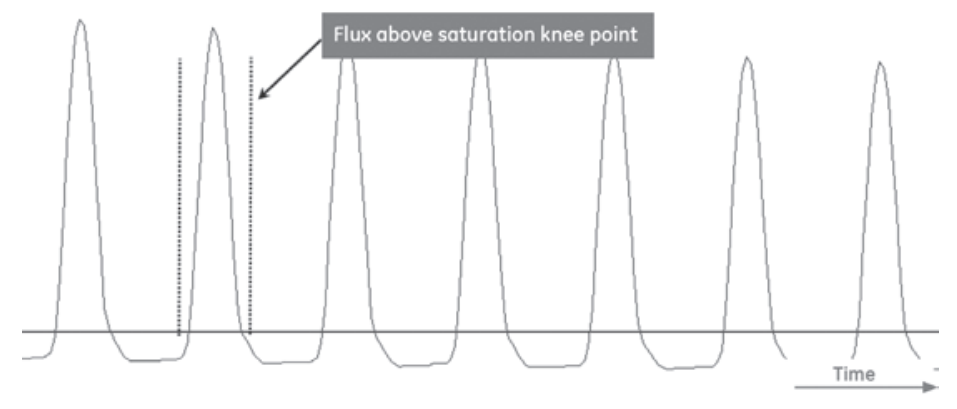

Figure 6.

Magnetizing Inrush Current Characteristic 


\subsection{Point on Wave}

The key factor in determining the magnitude of the magnetizing inrush current is the point on the voltage wave when the excitation voltage increases. If the excitation voltage is defined by

$$
\begin{aligned}
& e(t)=E_{\max } \cos (\omega t-\theta), \text { then the flux is defined by } \\
& \varphi(t)=\varphi_{\max } \sin (\omega t-\theta)+\varphi_{\max } \sin (\theta) .
\end{aligned}
$$

Where e(t) = instantaneous excitation voltage,

$E_{\text {max }}=$ peak exciting voltage,

$\omega \quad=$ system frequency,

$\theta \quad=$ voltage angle $\theta$ defined in Figure 7 ,

$\varphi(t) \quad=$ instantaneous flux,

$\varphi_{\max }=$ peak flux.

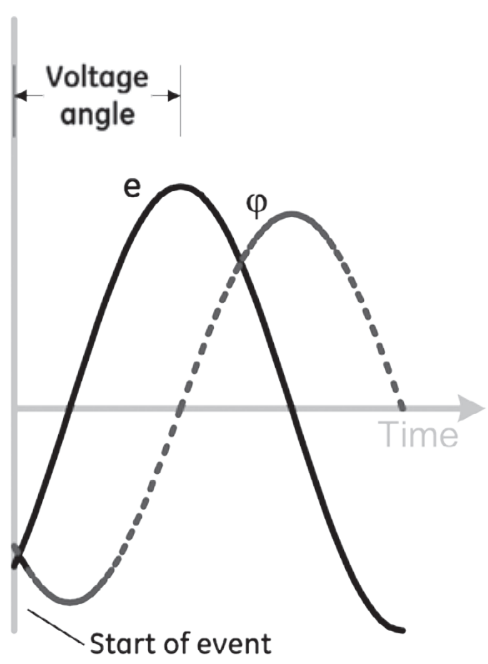

Figure 7.

Voltage Angle during Magnetizing Inrush

It is obvious that the flux is offset with respect to the 0-axis based on the voltage angle $\theta$. When the voltage angle $\theta$ is $90^{\circ}$, the flux is fully offset. There is no offset when the voltage angle $\theta$ is $0^{\circ}$. Maximum saturation of the transformer core occurs when the flux is fully offset at the $90^{\circ}$ voltage angle. [3]

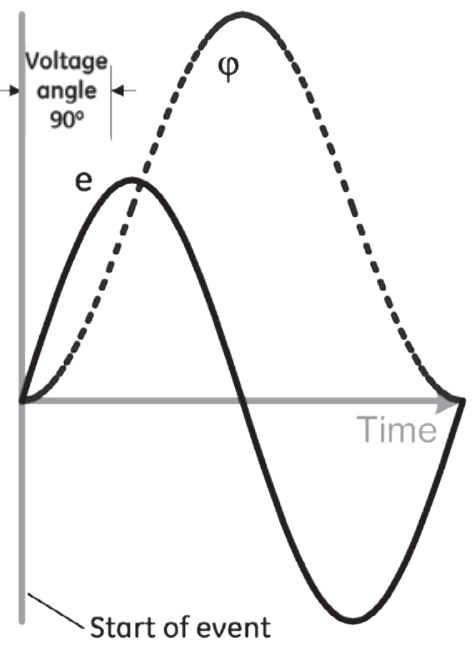

Figure 8.

Exciting voltage and flux at $90^{\circ}$ voltage angle

\subsection{Remanent Flux}

When a transformer is de-energized, some level of flux remains in the transformer core. This level of remanent flux is the flux in the core when the exciting voltage is removed. The actual value of the flux is based on the alignment of the magnetic moments of the steel, and can be found from the magnetic hysteresis loop of the transformer core. The remanent flux may therefore be positive or negative in value, and is typically $30 \%$ to $80 \%$ of the maximum flux of the core. When the transformer is energized, this remanent flux is added to the flux driven by the exciting voltage. The flux equation therefore becomes

$\varphi(t)=\varphi_{\max } \sin (\omega t-\theta)+\varphi_{\max } \sin (\theta)+\varphi_{\text {remanent }}$

, where $\varphi_{\text {remanent }}$ is the remanent flux in the core.

The flux characteristic during an inrush event is then offset with respect to the 0-axis. Depending on the sign of the remanent flux, the transformer core may be more or less likely to go into saturation. The impact of the remanent flux is removed once the core is fully saturated.

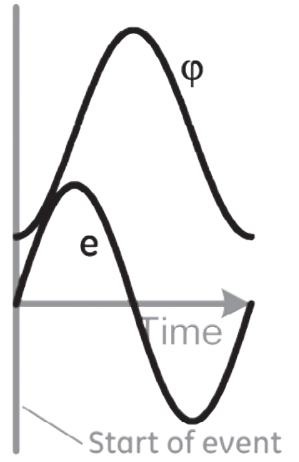

Positive remanent flux

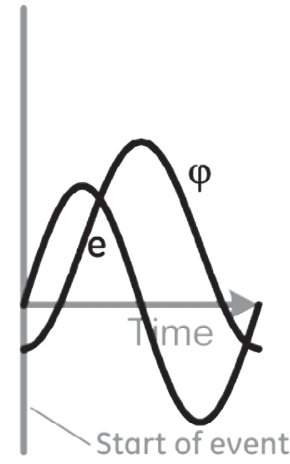

Negative remanent flux
Figure 9.

Excitation Voltage and Flux at $90^{\circ}$ Voltage Angle with Remanent Flux

\subsection{Transformer Design and Magnetizing Inrush Current}

The design of the power transformer influences the likelihood that the transformer core will saturate during inrush events. A transformer core is built from thin strips of high-grade electrical steel called laminations. The laminations are electrically isolated by a thin coating of insulation, and then stacked or wound to create the core section. The flux density of the steel, the design of the core, and the method of connecting the laminations all impact the amount and characteristics of the magnetizing inrush current.

Over the last few decades, there are some changes in transformer design that impact the second harmonic ratio during magnetizing inrush. The standard transformer design typically uses M- 6 conventional grain-oriented electrical steel. M-6 steel has a saturated flux density of 1.8 Teslas, the highest of any magnetic material. This very magnetically efficient steel results in lower exciting currents and therefore lower inrush 
currents. However, this has been the standard core material in transformer design for many years. Some transformers are now designed using high-permeability (High-B) electrical steel. High-B steels provide more consistent grain orientation, resulting in a more linear magnetic hysteresis loop.

A more important change has been in the construction of the core. Laminations were stacked on top of each other, resulting in an air gap between each lamination. The air gap increases the reluctance of the core, thereby reducing the magnetic efficiency of the core. Laminations are now constructed such that they overlap each other to provide a continuous path for the flux. This construction reduces the reluctance in the core, and therefore increases the flux density and reduces the exciting current.

The other significant change in transformer design is based around the economic concerns of loss evaluation. The trend is to select transformers based on loss evaluation. To limit losses, transformers are designed with lower maximum flux densities. The flux density is limited by using a core with a larger crosssectional area. The relation between maximum flux density and the exciting current is given by the following equation:

$$
E_{r m s} I_{\varphi, r m s}=4.44 f A_{c} l_{c} B_{\max } H_{r m s}
$$

$$
\text { Where } \begin{array}{ll}
\mathrm{E}_{\mathrm{rms}} & =\text { excitation voltage (rms) } \\
\mathrm{I}_{\varphi, \mathrm{rms}} & =\text { exciting current (rms) } \\
f & =\text { system frequency } \\
\mathrm{A}_{\mathrm{c}} & =\text { cross-sectional area of the core } \\
\mathrm{l}_{\mathrm{c}} & =\text { length of core path } \\
\mathrm{B}_{\max } & =\text { maximum flux density } \\
\mathrm{H}_{\mathrm{rms}} & =\text { permeability of the core (rms) }
\end{array}
$$

If the excitation voltage is constant, then a transformer with a lower flux density has a lower level of exciting current. Reductions in the required exciting current lead to a reduction in the magnetizing inrush current.[4] So the combination of efficient transformer core steel, better construction of the core, and the limiting of the maximum flux density, leads to lower exciting currents and lower magnetizing inrush currents.

\subsection{Power System Impedance}

The physical installation of the transformer also influences the magnetizing inrush current. The exciting voltage at the transformer is the system source voltage minus the voltage drop across the system impedance. As the source impedance decreases, indicating a stronger source, the magnitude of the inrush current increases. The resistance of the system is also a major contributor to the decay of the inrush current over time. The change in flux over time is defined by

$$
\Delta \varphi=\int_{t}^{t+T}(R \times i) d t
$$

where $\Delta \varphi \quad=$ flux change per cycle,

$\mathrm{R}=$ = total series resistance including transformer winding resistance

$\mathrm{T}=$ period of one cycle.

The flux in the transformer due to the inrush event begins to decay immediately by this amount, and decays until steadystate magnetizing flux is reached. As the flux controls the magnetizing current, the current also decays to steady-state magnetizing levels.[5]

\subsection{The Characteristics of Transformer Inrush Current}

As previously described, the classic inrush restraint current is similar to that of Figure 10. The non-linear nature of the magnetizing inrush current results in harmonics being present. It is possible to estimate the level of these harmonics by using Fourier series analysis on the magnetizing inrush current. The flux is above the saturation knee point for a total angular span of $2 \alpha$ radians. During this span, the exciting current is a portion of a sine wave. During the rest of the power system cycle, the exciting current is essentially 0 .

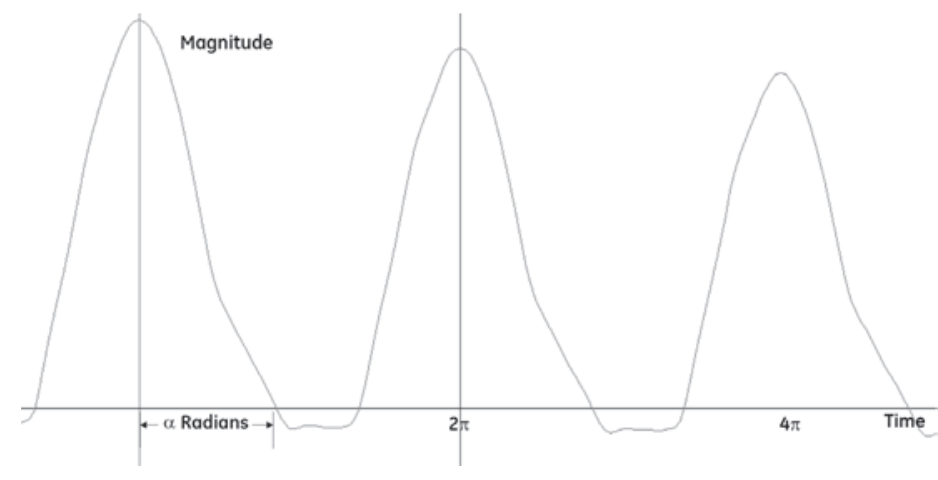

Figure 10.

Inrush Current Characteristic

This Fourier analysis shows that the second harmonic is the predominant harmonic during transformer inrush events and is commonly used as the basis for inrush restraint functions. As the saturation angle a increases, the exciting current becomes more linear and the ratio of second harmonics to fundamental decreases.[3] This means, in effect, the more severely the transformer core is saturated, the more linear the magnetizing inrush current.

Setting the midpoint of the first peak to time $t=0$, a cosine Fourier series may be used to calculate the second harmonic current and fundamental frequency component. Assuming the exciting current is truly symmetric, then the second harmonic ratio will be as high as $70.5 \%$ when $\alpha=\pi / 3$ radians, and will be $17.1 \%$ when $\alpha=2 \pi / 3$ radians.[3] 


\subsection{Summary of Transformer Inrush Phenomena}

Transformer inrush occurs whenever the excitation voltage on the transformer increases. Increasing the excitation voltage increases the flux in the transformer core, and therefore requires more current from the system to supply the flux. The new level of flux, and the period of the power system cycle the transformer core is in saturation determine the characteristics of the inrush current. The flux is offset based on the point on the voltage wave when the change in excitation occurs, and directly influences the level of saturation of the transformer core. Remanent flux in the core also initially offsets the flux in the core. The design and location of the transformer also impact the amount of saturation of the transformer core.

Of more interest for protection purposes are the characteristics of transformer inrush current. The common techniques for preventing the operation of differential elements for inrush events use the linearity of the differential current signal. The ratio of second harmonic current to the fundamental current is often used. The more linear the inrush current, the less second harmonic current is present. Therefore, as the level of core saturation increases, the ratio of second harmonic current to fundamental current decreases.

\section{Events that result in Magnetizing Inrush Currents}

Any event on the power system that causes a significant increase in the magnetizing voltage of the transformer core results in magnetizing inrush current flowing into the transformer. The three most common events are:

Energization of the transformer. This is the typical event where magnetizing inrush currents are a concern. The excitation voltage on one winding is increased from 0 to full voltage. The transformer core typically saturates, with the amount of saturation determined by transformer design, system impedance, the remanent flux in the core, and the point on the voltage wave when the transformer is energized. The current needed to supply this flux may be as much as 40 times the full load rating of the transformer, with typical value for power transformers for 2 to 6 times the full load rating.[1] The waveforms of Figure 3 were recorded during energization of a transformer.

Magnetizing inrush current during fault clearing. An external fault may significantly reduce the system voltage, and therefore reduce the excitation voltage of the transformer. When this fault is cleared, the excitation voltage returns to the normal system voltage level. The return of voltage may force a dc offset on the flux linkages, resulting in magnetizing inrush current. This magnetizing inrush current will be less than that of energization, as there is no remanent flux in the core.[3] The current measured by the differential relay will be fairly linear due to the presence of load current, and may result in low levels of second harmonic current.
Sympathetic inrush. Energizing a transformer on the power system can cause sympathetic magnetizing inrush currents to flow in an already energized parallel transformer. Energizing the second transformer causes a voltage drop across the resistance of the source line feeding the transformers. This voltage drop may cause a saturation of the already energized transformer in the negative direction. This saturation causes magnetizing inrush current to supply the flux. The magnitude of the magnetizing inrush current is generally not as severe as the other cases.[3][5][6][6]

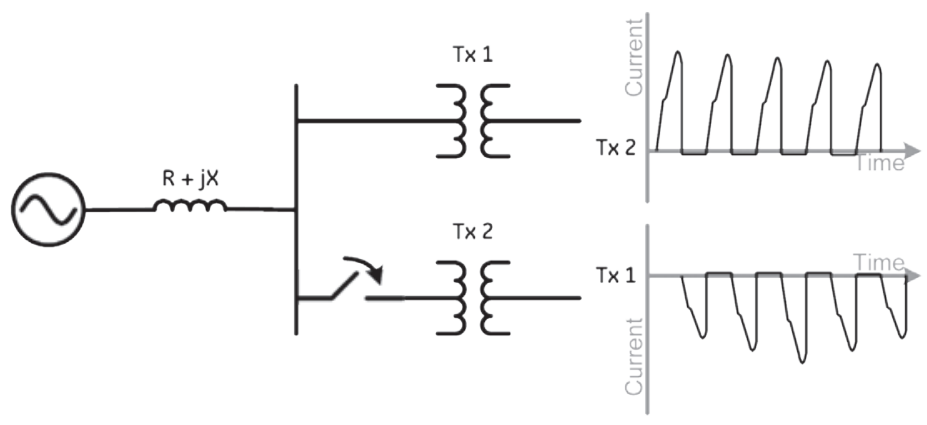

Figure 11.

Sympathetic Inrush Circuit and Waveforms

\section{Transformer Inrush Restraint Methods for Differential Protection}

Transformer inrush restraint functions are intended to block the differential element from operating during such an inrush event and permit the differential element to operate for internal fault events. The challenge, obviously, is that inrush current and an external fault both present a large differential current to the differential element. There are many different methods that have been proposed and implemented for restraining the differential element during a transformer inrush condition. These methods are discussed in a paper presented at this conference in 2000.[7]

FPL has already decided on a specific model of transformer differential relay for their standard protection of transformers at the transmission level. Therefore, this paper discusses only the options for inrush restraint available in this model of relay. This paper also discusses the choice of inrush restraint mode. The restraint mode determines if inrush restraint is applied on a per-phase or multi-phase basis. The inrush restraint methods available to FPL in their chosen relays are the harmonic restraint and adaptive harmonic restraint functions.

\subsection{Harmonic Restraint}

Harmonic restraint is the classical way to restrain tripping. There are many variations on this method. All of these methods work on the assumption the magnetizing inrush current contains high levels of second harmonic current. The current for an internal transformer fault typically has very low levels of second harmonic current. The simplest method of harmonic restraint 
uses the magnitude of the second harmonic in the differential current compared to the magnitude of the fundamental frequency component in the differential current. Tripping of the differential element is blocked when this ratio exceeds an adjustable threshold.

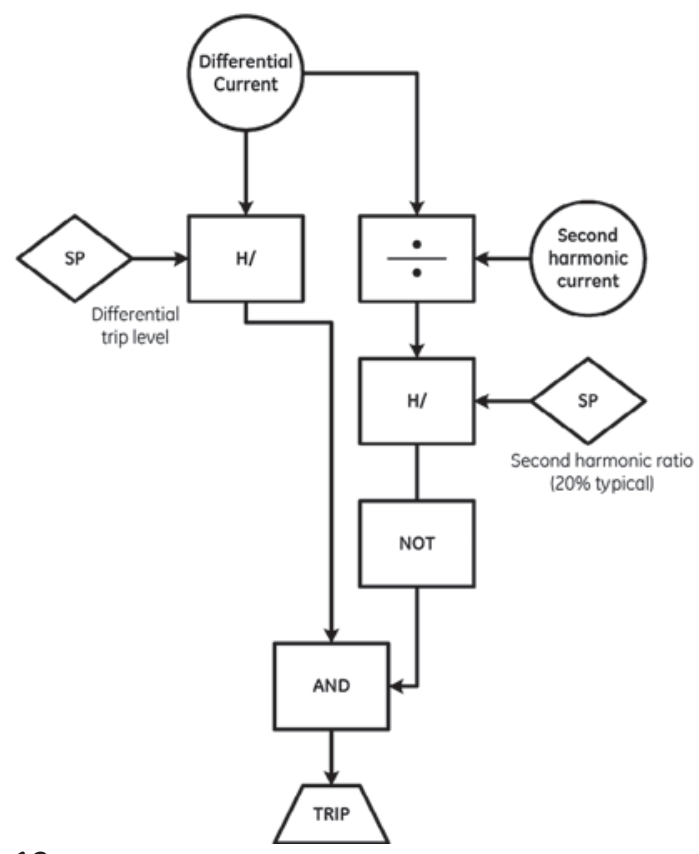

Figure 12.

SAMA Diagram for Second Harmonic Restraint

In this paper, the term "second harmonic ratio" is defined as:

$$
\frac{I_{\text {Differential (2 }} \text { nd harmonic) }}{I_{\text {Differential ( fundamental) }}}
$$

This method originated in electromechanical relays, and has been carried through as the most common method in microprocessor relays. The harmonic restraint is typically calculated on a per-phase basis. Variations include using the RMS current as opposed to the fundamental frequency component, and using a cumulative three-phase implementation.

The historical setting for harmonic restraint is a second harmonic ratio of $20 \%$, with an available setting range of $1 \%$ to $40 \%$. Set too high, and the differential element may trip during transformer energizing. Set too low, and inrush restraint may block tripping during some internal fault events.

\subsection{Adaptive Harmonic Restraint}

Adaptive harmonic restraint is a modified version of traditional harmonic restraint that considers the magnitude and phase of the second harmonic and fundamental frequency component in the differential current. Some inrush events initially produce low levels of second harmonic in the differential current, as in the example of Figure 13. This phenomenon is an indication the remanent flux in the core initially pushes the core deeper into saturation. This low level of second harmonic current may allow the differential element to operate.

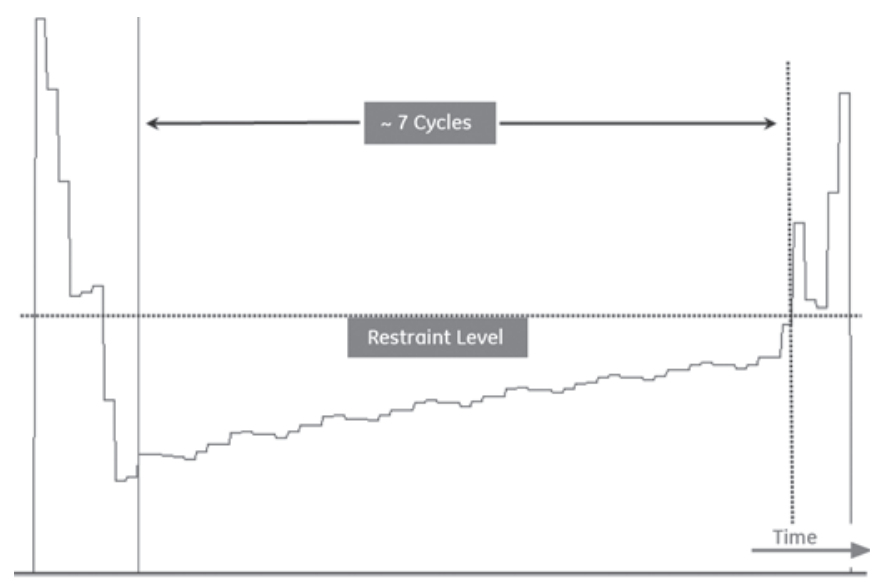

Figure 13.

Second Harmonic Current During Inrush

The adaptive harmonic restraint method dynamically changes the inrush restraint level to properly restrain the differential element for these cases. This method uses the discriminating signal:

$$
\vec{I}_{21}=\frac{\vec{I}_{2}}{\vec{I}_{1} \times e^{j \omega t}}
$$

where $\quad \vec{I}_{2}$ is the second harmonic differential current phasor

$\vec{I}_{1} \quad$ is the fundamental differential current phasor, and (1) is the system frequency.

The phase angle of the discriminating signal is always $90^{\circ}$ or $270^{\circ}$ for an inrush condition. Consider a typical harmonic restraint threshold of $20 \%$, as plotted on the polar graph of Figure 14. The adaptive harmonic restraint initially has a lower inrush restraint threshold along the $90^{\circ}$ and $270^{\circ}$ axes. This threshold is dynamically raised to the default setting of $20 \%$ over a period of 5-6 cycles.[8]

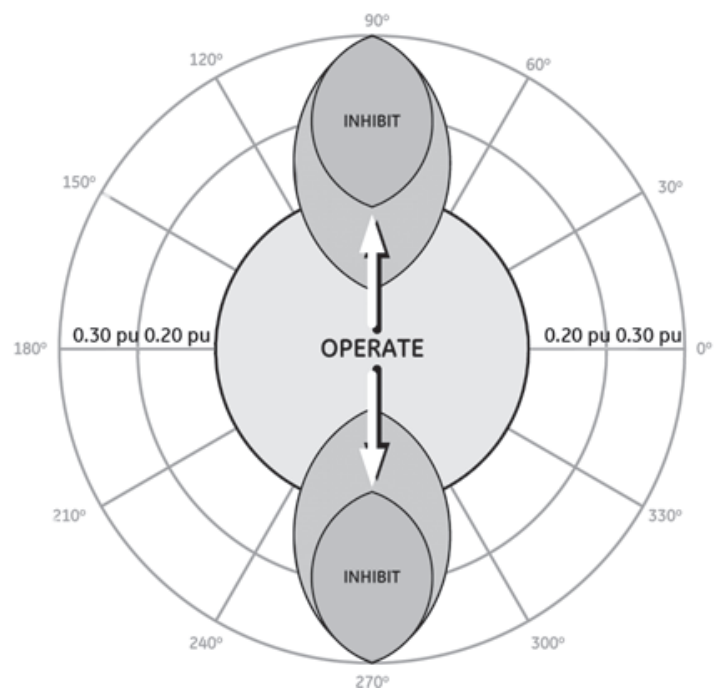

Figure 14

Adaptive Harmonic Restraint Characteristic

The adaptive harmonic restraint algorithm successfully restrains tripping when faced with low levels of second harmonic current during an inrush event. However, this algorithm may slow tripping of the differential element by a few cycles for an internal fault if some second harmonic is present in the current. 


\subsection{Inrush Restraint Mode}

Harmonic restraint and adaptive harmonic restraint are normally calculated individually on each phase. Typically, the operation of the differential element on any phase operates the circuit breakers supplying the transformer. If the restraint criterion is not met on any phase, the transformer may be tripped offline during energization or sympathetic inrush. Depending on the transformer installation, operating requirements, and operating philosophy, this may be acceptable performance of the differential element. However, for the standard FPL application, it is more desirable to increase the security of the differential element against inrush events by looking at inrush restraint functions in more than one phase. The inrush restraint mode is therefore the method of implementing the inrush restraint function across the entire transformer.

Per-phase. In per-phase mode the relay performs inrush restraint individually in each phase.

2-out-of-3. In 2-out-of-3 mode, the relay checks second harmonic level in all three phases individually. If any two phases establish a blocking condition, the remaining phase is restrained automatically.

Averaging. In averaging mode, the relay first calculates the average second harmonic ratio, and then applies the inrush threshold to the calculated average.

1-out-of-3. In 1-out-of-3 mode, all three phases are restrained when a blocking condition exists on any one phase. 1-out-of-3 mode typically reverts back to per-phase mode after a short time delay to allow tripping in case an internal fault occurs during energization.
These restraint modes may be explicitsettings of the transformer differential element. They may also be implemented in the flexible configuration logic of the transformer protection relay.

The transformer differential protection operating for an inrush event is a loss of security. These inrush restraint modes are listed in order from the least secure to the most secure. Comparing these restraint modes to an actual transformer energization event can provide some illustration of performance. For this event, the magnetizing inrush currents during energization of the transformer were high enough to cause operation of the differential element. The second harmonic current ratios are shown in Figure 15. The B-Phase ratio is greater than 1 in this case, and is not shown on the graph. This differential element uses traditional harmonic restraint set at $20 \%$ for the inrush restraint mode. Tripping on any phase de-energizes the transformer.

Table 1 lists the performance of the various restraint modes for this example. Per-phase mode will allow the differential to operate, while all the other modes will block the differential. The correct choice is a matter of application, and a matter of operating philosophy. Per-phase mode may be the most appropriate solution for a three-phase bank made up from single-phase transformers, for example.

\begin{tabular}{|l|l|}
\hline Restraint Mode & Result \\
\hline Per-phase & Differential trips on A-phase \\
\hline 2-out-of-3 & Differential restrains: B-phase and C-phase blocked \\
\hline Averaging & Differential restrains: at $t=0,=(0.05+6.12+0.29) / 3=2.15$ \\
\hline 1-out-of-3 & Differential restrains: B-phase and C-phase blocked \\
\hline
\end{tabular}

Table 1.

Restraint Mode Results

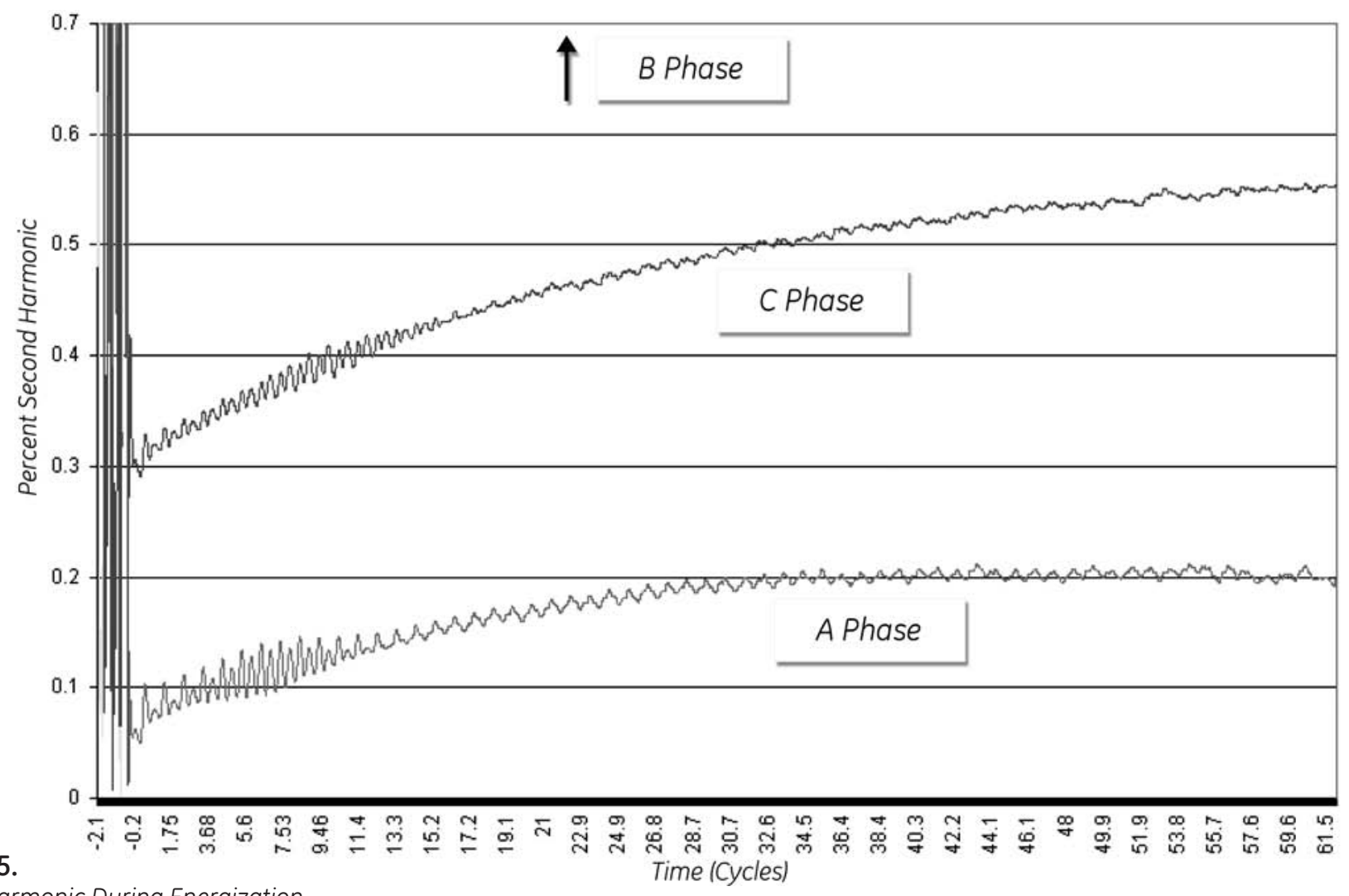

Figure 15.

Second Harmonic During Energization 


\section{FPL Testing of Inrush Restraint Methods}

Ideally, setting the inrush restraint function for the transformer differential element is exactly like setting any other protection function. The key criteria are sensitivity and selectivity. The setting must be sensitive enough to recognize magnetizing inrush current even with low levels of second harmonics. And it must be selective to distinguish between an inrush event and other events that may produce second harmonic current.

The standard process for setting a protection function is to perform an analysis of the system. This analysis models the response of the system for various transient events to provide a basis for setting protection functions. For example, to set a distance element, the first step is to perform a short circuit analysis. The short circuit analysis is built around the known quantity of the system impedances. Different predictable scenarios are used during the analysis; basically system operating contingencies and fault location. Then some educated guesses are used for the influence of unknown variables, such as fault resistance.

A similar process can be identified for setting the transformer inrush restraint function but the results are not as predictable as short circuit analysis. The known quantity for inrush restraint analysis is that of transformer design and transformer location. There isn't any "predictable" scenario for modeling the inrush. There is only the ability to make educated guesses that relate to the type of event that causes inrush, the point on the voltage wave when this event occurs, and the amount of remanent flux in the core of the transformer. And unlike the influence of fault resistance on a short circuit analysis, there is no empirical model of a transformer for the influences of some of these factors.

Therefore there is no empirical method for determining the magnitude of inrush currents and the second harmonic ratio in the differential current. FPL used a model of the system and transformer to produce some digital representation of inrush events, and also used oscillography captured during transformer inrush events to develop settings using an experimental process.

A protective relay that FPL has selected for use in the transformer protection panel provide the following options:

Inrush restraint function: harmonic restraint adaptive harmonic restraint

\section{Inrush restraint mode: per-phase \\ 2-out-of-3 \\ averaging}

In addition, the 1-out-of-3 inrush restraint mode can be implemented using the flexible configuration logic of the relay.

This test procedure uses wye-connected CTs that are typical on new installations using microprocessor-based differential relays. Delta-connected CTs complicate inrush restraint settings. The currents measured by the relay are phase-phase currents. The subtractive effect of the delta connection may actually decrease the second harmonic current seen by the relay and require a lower setting on the inrush restraint function.[9]

Therefore, the concern for setting the inrush restraint function is the ratio of the second harmonic current to the fundamental current. Having some guidelines that predict this ratio will help develop inrush restraint settings that are sensitive and selective.

Figure 16.

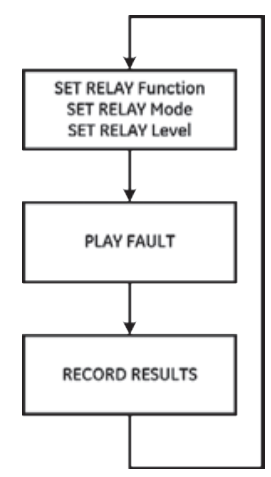

FP\&L Test Procedure

To determine the most appropriate choice for the inrush restraint method and the inrush restraint mode, a simple bench test experiment was devised. The procedure was to simply choose some settings for the relay, connect the relay to a three-phase test set, and play several oscillography files through the relay.

The oscillography file of Figure 17 shows a misoperation of a differential relay caused by low levels of second harmonic current during energization of an autotransformer. This transformer is a bank of 3 single-phase 500MVA, 500kV/230kV autotransformers, and was energized from $500 \mathrm{kV}$.

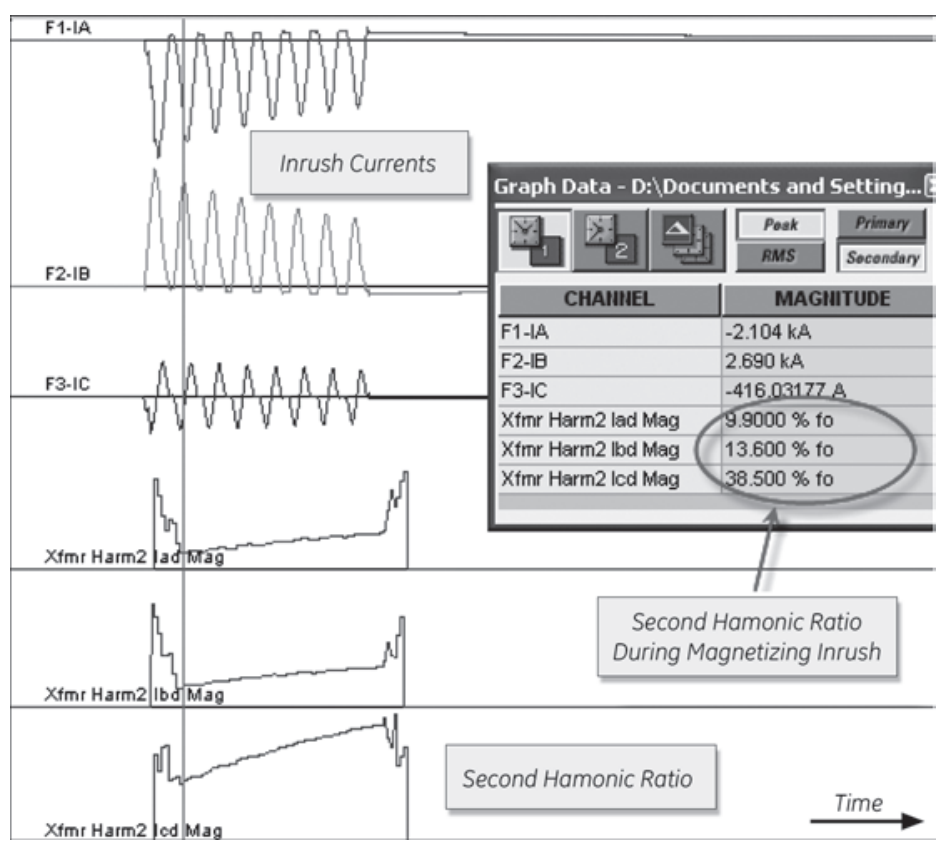

Figure 17.

Oscillography File used for Test Procedure 
Before beginning the testing, FPL set the following criteria for acceptable settings.

- A minimum level setting for harmonic restraint of $15 \%$.

- No operating time delay for the differential element is introduced by the selected inrush restraint function or inrush restraint mode.

- The differential element is blocked from operating during this actual energization event.

Based on these criteria, the adaptive harmonic restraint function and the 1-out-of-3 restraint modes were not tested. The adaptive harmonic restraint function could possibly slow tripping of the differential element for internal faults where CT saturation could occur. The 1-out-of-3 mode must be implemented in the flexible configuration logic of the relay. The concern is this logic may introduce a time delay when blocking the differential element. Also, FPL would like to avoid custom logic for protection functions as much as practical.

\subsection{Test Results}

The more detailed test procedure is shown in Figure 18. The initial setpoint for the inrush restraint level was 20\%. If the differential element tripped at this level, the level was then reduced to $15 \%$. If the differential element continued to trip, the level was reduced until restraint was achieved. The results of the test are tabulated in Table 2.

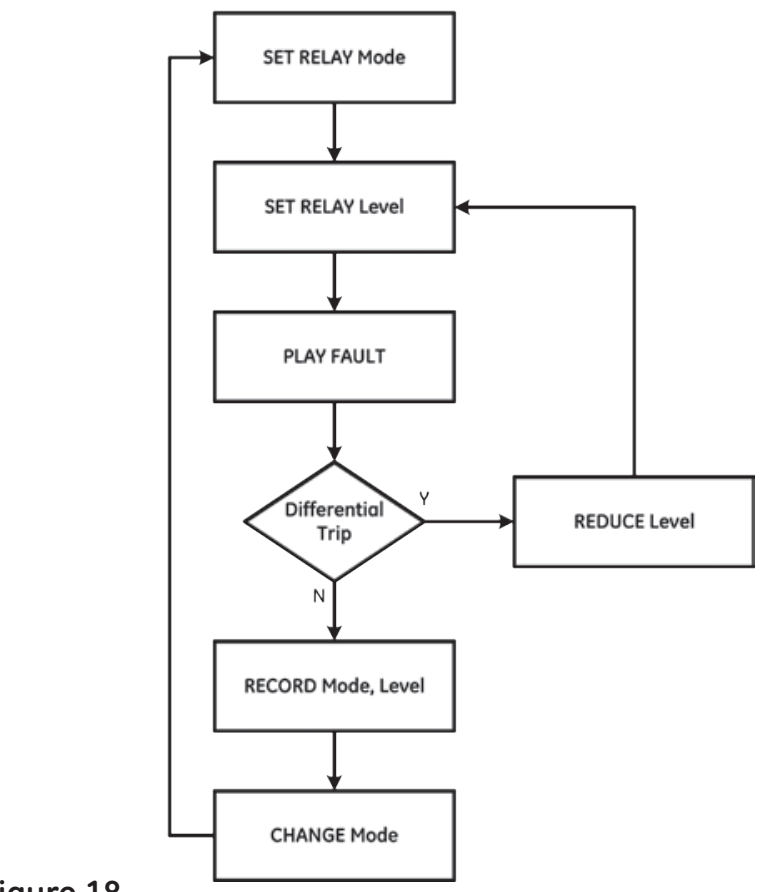

Figure 18.

Detailed Test Procedure

These results match the expected results for the different inrush restraint modes. No level was recorded for the per-phase option, as a review of the fault data indicated the level would be $10 \%$ or less. This is an unacceptably low setting. Using the averaging mode, the differential element was blocked at 20\%. The inrush restraint level was raised until a trip occurred to give some idea of the margin between blocking and tripping for the differential element.

\begin{tabular}{|l|l|}
\hline Mode & Level to achieve blocking \\
\hline Per-phase & No level recorded \\
\hline 2 -out-of-3 & $13 \%$ (Trips at $14 \%$ ) \\
\hline Averaging & $20 \%$ (Trips at $21 \%$ ) \\
\hline
\end{tabular}

Table 2.

Harmonic Restraint Test Results

\section{Conclusions}

The traditional settings for inrush restraint for transformer differential protection are to use harmonic restraint in a perphase mode, with a restraint level of $20 \%$. Experience shows that for most transformer protection applications these settings provide high-speed clearing of transformer faults and proper restraint for inrush events. For a few applications, lowering the harmonic restraint setting, employing cross-blocking techniques, or a combination of both may be necessary to ensure that transformer energizations occur successfully where very low second harmonic inrush levels are present. Capturing energization records to confirm where these techniques may need to be employed is essential.

Based on operating experience, FPL has some transformer protection applications where the traditional inrush restraint settings are not adequate. For some of these applications, FPL plans to use either 2-out-of-3 or averaging mode, to provide greater security. A setting of $15 \%$ provides a good margin of security for energization of the transformer where the second harmonic current may fall to low levels. The belief is these settings will also successfully restrain the differential element when faced with a fault recovery magnetizing inrush event.

There may be some concern when setting the inrush restraint level to $15 \%$ or lower. CT saturation during internal faults may result in the protection relay seeing a high second harmonic current ratio and incorrectly restraining. Setting an unrestrained differential element between 8 per unit and 10 per unit provides confidence the transformer protection will trip for an internal fault even at a lower setting for the inrush restraint.

FPL has used this process to guide the development of inrush restraint settings. The limited experience to date with intelligent consideration of the settings has been successful. The following event records are from two different transformer locations that used these settings. The transformers in both cases had previously been energized, so remanent flux was present in the transformer core. Figure 19 is for a 560MVA, 230kV/138kV three-phase autotransformer, energized at $230 \mathrm{kV}$. Figure 20 is for a $224 \mathrm{MVA}, 230 \mathrm{kV} / 138 \mathrm{kV}$ autotransformer, energized at $230 \mathrm{kV}$.

Setting the inrush restraint function takes some knowledge and experience. The transformer design and the system impedance have some influence on the magnitude and severity of the inrush event. Capturing oscillography data for every energization event for a specific transformer may be used to generalize about the characteristics of inrush currents for a specific transformer. If a transformer seems to provide low levels of second harmonic 


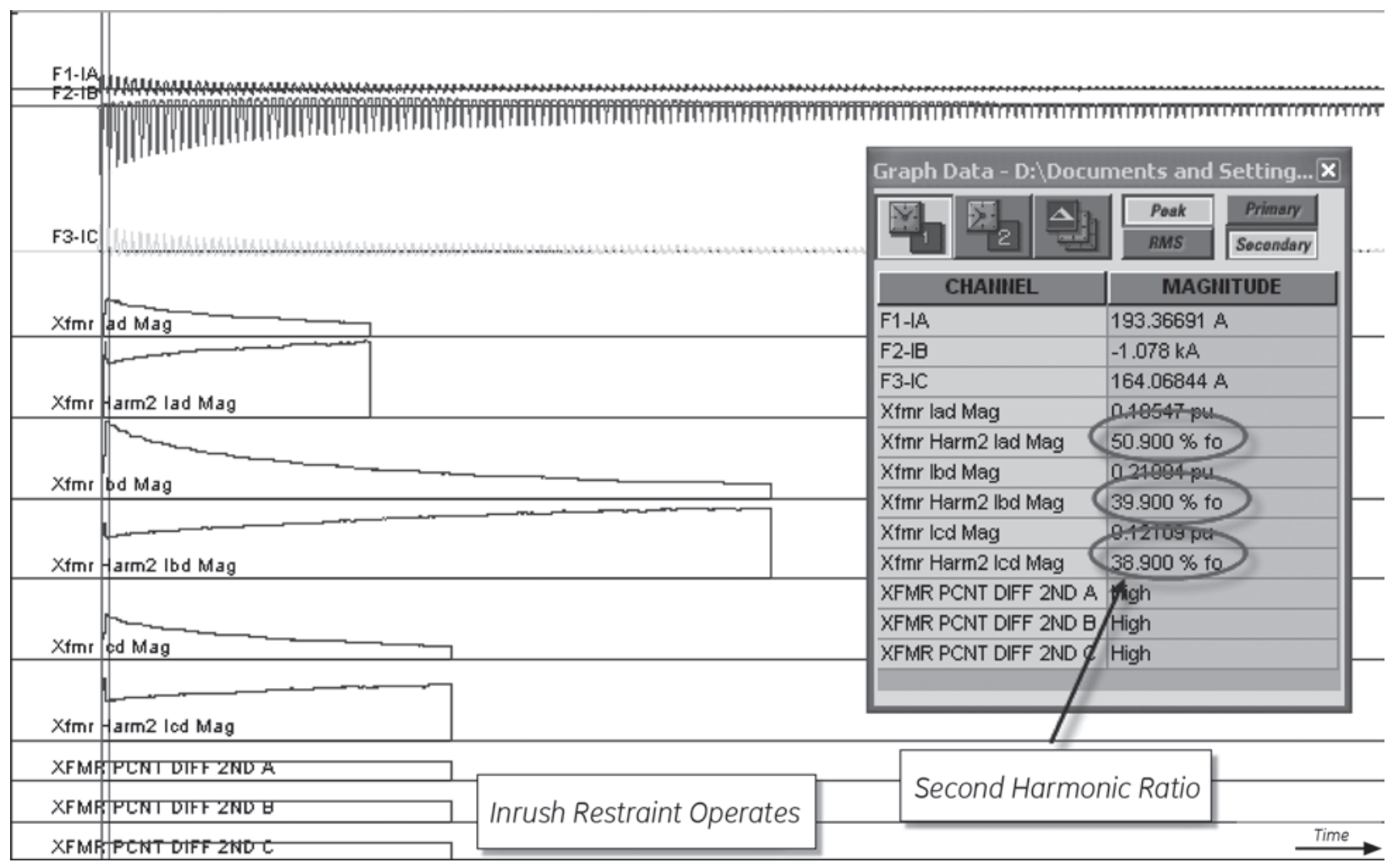

Figure 19.

Energization of One Autotransformer

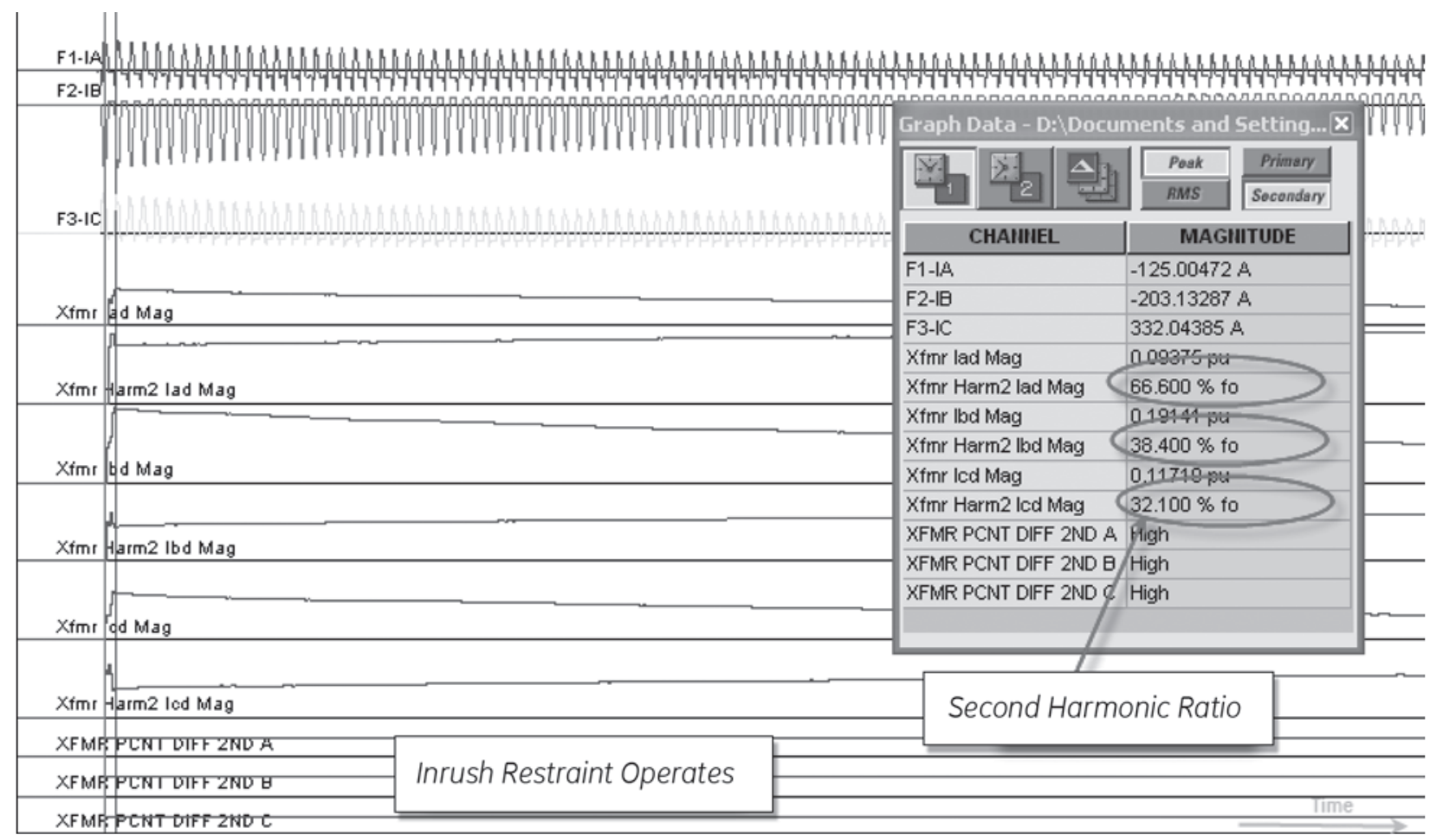

Figure 20.

Energization of a Second Autotransformer 
current over a number of events, it may be necessary to lower the inrush restraint functions or to use adaptive harmonic restraint. FPL plans to capture and analyze oscillography data on every energization of a specific transformer to develop the operating history for a specific transformer.

\section{Symbols}

The process control industry has developed symbols and diagramming formats to represent both linear and non-linear processes. The symbols and diagramming format are commonly known as "SAMA diagrams", as they were originally a standard developed by the Scientific Apparatus Makers Association. Though the Scientific Apparatus Makers Association has declared the original standard obsolete, and no longer permits the direct association of the organization name with the industry standard, these symbols, and the term "SAMA diagram", are still in common use in the process control industry. These symbols will grow increasingly useful as protection systems migrate from traditional protection and control to automatic process control.

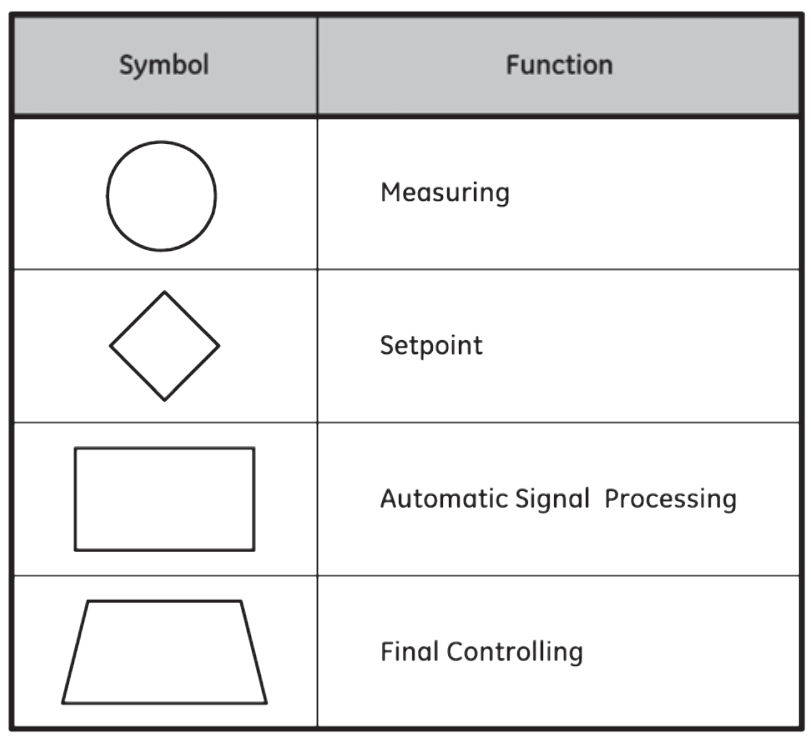

\begin{tabular}{|c|l|}
\hline Symbol & \multicolumn{1}{|c|}{ Function } \\
\hline $\mathrm{H} /$ & High signal monitor \\
\hline$\bullet$ & Dividing \\
\hline NOT & Inverter \\
\hline AND & Logical AND \\
\hline
\end{tabular}

\section{References}

[1] L. L. Grigsby, editor, "The Electric Power Engineering Handbook", CRC Press LLC, Boca Raton, FL, 2001, p. 3-29.

[2] A. E. Fitzgerald, C. Kingsley, Jr., S. D. Umans, "Electric Machinery, 4th edition", McGraw_Hill Book Company, New York, NY, 1983, pp. 4-24.

[3] S. H. Horowitz, A. G. Phadke, "Power System Relaying, 2nd edition", Research Studies Press Ltd., Somerset, England, 1995, pp. 210-214.

[4] C. E. Lin, C. L. Cheng, C. L. Huang, J. C. Yeh, "Investigation of Magnetizing Inrush Current in Transformers Part I - Numerical Simulation", IEEE Transactions on Power Delivery, Vol. 8, No. 1, January, 1993, pp. 246-254.

[5] H.S. Bronzeado, P. B. Brogan, R. Yacamini, "Harmonic Analysis of Transient Currents During Sympathetic Interaction", IEEE Transactions on Power Systems, Vol. 11, No. 4, November 1996.

[6] M. M. Saied, "A Study on the Inrush Current Phenomena in Transformer Substation", Conference Record of the 36th Annual Meeting of the IAS, 2001. pp. 1180-1187.

[7] R.W. Patterson, W. P. McCannon, G. L. Kobet, "A Consideration of Inrush Restraint Methods in Transformer Differential Relays", 54th Annual Georgia Tech Protective Relaying Conference, Georgia Tech, Atlanta, GA, May 3-5, 2000.

[8] B. Kasztenny, A. Kulidjian, "An Improved Transformer Inrush Restraint Algorithm Increases Security While Maintaining Fault Response Performance", 53rd Annual Conference for Protective Relay Engineers, Texas A\&M, April 11th - 13th, 2000.

[9] G. L. Kobet, R. W. Patterson, "Matlab Analysis of Braytown Transformer Differential Inrush Misoperation", Georgia Tech Fault and Disturbance Analysis Conference, Atlanta, GA, May 1-2, 2000.

\section{Bibliography}

[B1] C. E. Lin, C. L. Cheng, C. L. Huang, J. C. Yeh, "Investigation of Magnetizing Inrush Current in Transformers Part II - Harmonic Analysis", IEEE Transactions on Power Delivery, Vol. 8, No. 1, January, 1993, pp. 255-263.

[B2] GE Publication GEK-113327A, "T60 Transformer Management Relay", Instruction Manual, Markham, Ontario, Canada, 2006

[B3] G. Ziegler, "Numerical Differential Protection", Publicis Corporate Publishing, Erlangen, Germany, 2005. pp. 146151. 